\title{
Study of PSO-SVM Model for Daily Water Demand Prediction
}

\author{
Baiyi Jiang1, a , Tianwei Mu ${ }^{1, b, ~}$, Ming Zhao ${ }^{1, c}$, Danyu Shen ${ }^{1, d}$ and Lingping Wang ${ }^{1, \text { e }}$ \\ ${ }^{1}$ School of Municipal and Environmental Engineering, Shenyang Jianzhu University, Shenyang, \\ 110168, China; \\ ${ }^{2}$ School of Municipal and Environmental Engineering, Harbin Institute of Technology, Harbin, \\ 150090, China

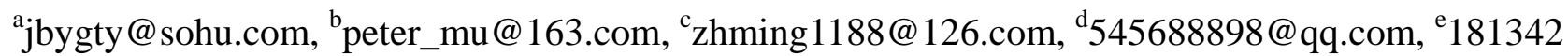 \\ 2699@qq.com
}

\begin{abstract}
For solving the problem of daily water demand in G city, a method of particle swarm optimization algorithm combined support vector machine (PSO-SVM) is presented. The expansion constant and penalty factor firstly are selected by particle swarm optimization (PSO). Secondly, the historical water demands data are trained by support vector machine (SVM). Finally, the new independent variables are employed to predict water demands in next time. By comparing BP and SVM with this method, the results show that the real and predicted daily water demands errors are less than the other models. Therefore, this method is a effective way to predict daily water demands of city $\mathrm{G}$.
\end{abstract}

Keywords: Particle swarm optimization, support vector machine, Daily water demand prediction

\section{Introduction}

Nowadays, with the development of economy, the water demands of urban residents are increasing rapidly. For meeting the requirement of customers, statistics, analysis and prediction for water demands should be made from water supply departments. Water demand prediction are divided into three parts, including short, middle and long prediction. Daily water demand prediction belong to the short and time series model frequently is used consequence of simple, fast and well-adapted. According to the historical data series, water demands of next time are predicted in time series model [1]. However, too many factors should be considered in short predicted, which may affect the precision of prediction. So, the regression analysis model is used to analyze historical data by factors of influence, which employs quantitative management equation to demonstrate the relationship of factors and water demands [2]. Thus, Building the regression equation can improve effectiveness of the short predicion.

A large number of regression models are employed to predict the water demand. Jiang et al.(2008), considering season, weather and festival, successful solve the problem of daily water demand by BP neural network model [3], but this model rely too heavily on trained data set. If the data set is too much, the over-fit phenomena will appear that decreases the precision. Zhao et al.(2012) employed the SVM model [4], avoiding the over-fit and improve the generalization. However, the speed of this model are slower than BP network. Li et al.(2016) employed Grey Markov model [5], analyzing the factors undefined problem of water demand systemically. It improve the generalization and speed, but the precision less than SVM model. 
In conclusion, for a better prediction a case of city G, based on PSO algorithm [7], the SVR model is used to predict daily water demand.

\section{PSO-SVM Model}

SVM model transfer nonlinear to linear equation and rise historical data dimension by kernel function. Finally, the optimal hyperplane is found, which make maximum empty spaces at two sides of coordinate. If set independent variable is $\vec{x}_{i}=\left(x_{i 1}, x_{i 2}, \ldots, x_{i n}\right)$ and water demand is $y_{i}$, the SVM model equation can be written as equation (1):

$$
\vec{q}^{\prime}=\frac{1}{2} \sum_{i, j}^{l} \alpha_{i} k\left(\vec{x}_{i}, \vec{x}_{j}\right)+\beta
$$

Where $\vec{q}^{\prime}$ is the series of water demand prediction; $k$ is kernel function, selected RBF, shown as equation (2); $\alpha_{i}$ is the undetermined parameters and the equation shown as (3); $\beta$ is offset value; $\vec{x}_{j}$ is independent variable series at time $j$ and $j \neq i$; $l$ is the number of independent variable.

$$
k\left(x_{i}, x_{j}\right)=e^{-\left\|x_{i}-x_{j}\right\| / 2 \sigma^{2}}
$$

Where $\sigma$ is the extension constant of the radial basis function.

$$
\alpha_{i}=C \xi_{i}
$$

Where $C$ penalty factor; $\xi_{i}$ is slack variable of $\alpha_{i}$.

PSO model is adopted to optimize $C$ and $\sigma$ and the process reference. In the trained process, the historical data is divided into 5 parts. 5-fold cross-validation (CV-5) is employed for calculating [6]. The minimum of mean absolute error (MAE) is used as objective of PSO, shown as equation (4). The parameters of $\alpha_{i}$ and $\beta$ can be solved at last.

$$
E=\sum_{i=1}^{V} \frac{1}{2} \sum_{t=1}^{T}\left(y_{t}-y_{t}^{\prime}\right)^{2}
$$

Where $E$ is calculation error; $q_{t}$ is the real water demand value at date $t ; q_{t}^{\prime}$ is the predicted water demand value at date $t ; V$ is the times of cross validation, $V=5$.

\section{Case Study}

The historical water demand data, including 850 training samples and 7 testing sample, from Jan 12014 to May 72016 are imported to Matlab 2016a. Using Matlab language, PSO-SVM model is programmed. The flowchart is shown in Fig. 1. 


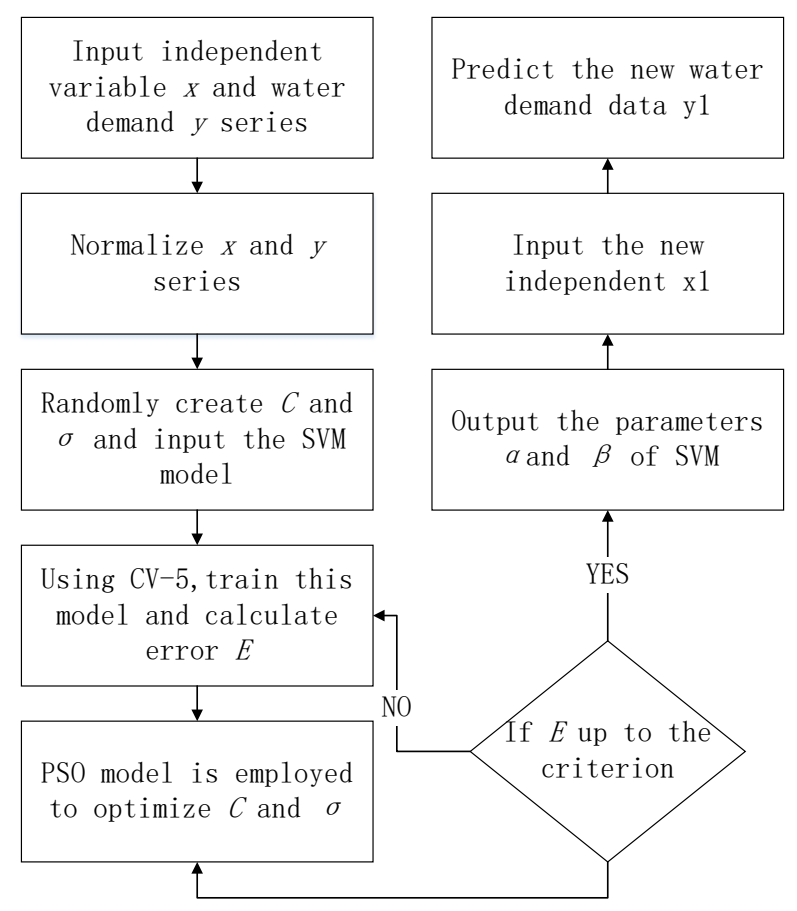

Fig. 1 Flowchart of PSO-SVM model

The water demand data of trained samples are inputted into Matlab, shown in Fig. 2.

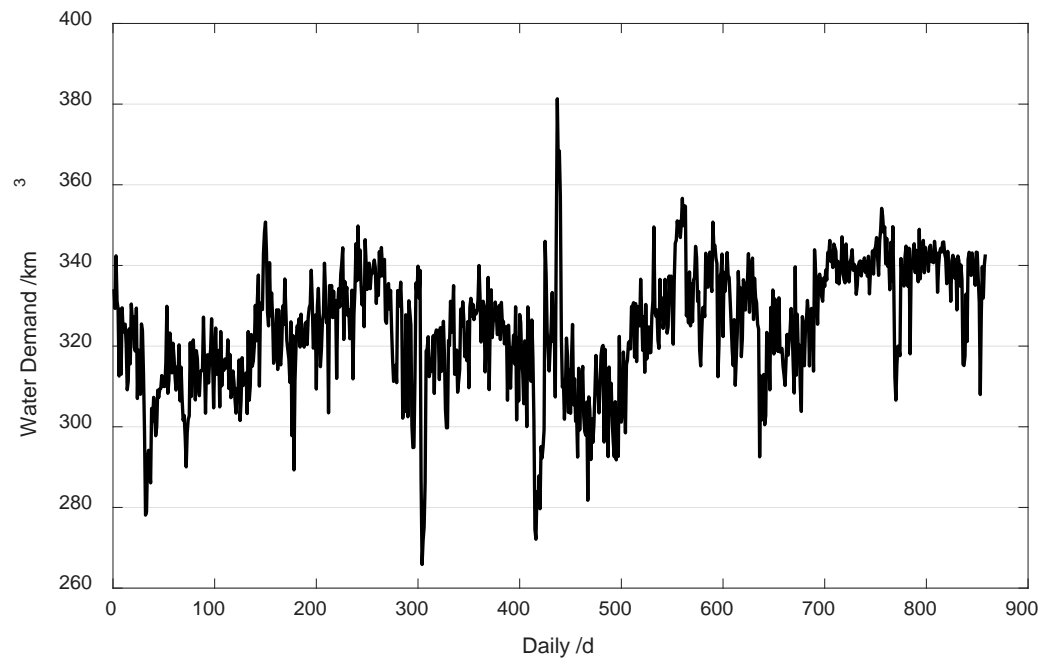

Fig. 2 Trained data of daily water demand

This paper consider daily maximum temperatures, daily minimum temperatures, week values, weather conditions (morning and evening), and wind scales (morning and evening) as independent variables and input into Matlab. For transferring independent variables to the forms of fitting PSO-SVM model, under the condition of retaining original value of temperature and week, digital processing is applied by forms of Table 1 and 2 . 
Table 1 Weather conditions

\begin{tabular}{|c|c|}
\hline Weather conditions & Digital values \\
\hline Sunny & 1 \\
\hline Haze & 2 \\
\hline Cloudy & 3 \\
\hline Shade & 4 \\
\hline Fog & 5 \\
\hline Light snow & 6 \\
\hline Snow shower & 7 \\
\hline sprinkle & 8 \\
\hline Shower & 9 \\
\hline Thunderstorm & 10 \\
\hline Moderate rain & 11 \\
\hline Blowing sand & 12 \\
\hline Rainstorm & 13 \\
\hline Sleet & 14 \\
\hline Moderate snow & 15 \\
\hline Floating dust & 16 \\
\hline Sprinkle to moderate rain & 17 \\
\hline Heavy rain & 18 \\
\hline Light to moderate snow & 19 \\
\hline
\end{tabular}

Table 2 Wind scale

\begin{tabular}{|c|c|}
\hline Wind scales & Digital values \\
\hline$<3$ & 0 \\
\hline $3 \sim 4$ & 1 \\
\hline $4 \sim 5$ & 2 \\
\hline $5 \sim 6$ & 3 \\
\hline $6 \sim 7$ & 4 \\
\hline
\end{tabular}

Secondly, these samples are normalized and the error of the penalty function in SVM is set as 0.0001 , and kernel function shown as equation (4). Randomly, create penalty function $C$ and extension constant $\sigma$ and input the SVM model. Adopting CV-5 divide data into five parts, and using PSO model calculate the sum of minimum of MAE in each part. The results show in Table 3.

Table 3 The result of water usages prediction

\begin{tabular}{|c|c|}
\hline$C$ & $\sigma$ \\
\hline 2.49 & 1.19 \\
\hline
\end{tabular}

These parameters are inputted to the SVM model, and the trained samples are trained. The parameters of $\alpha_{i}$ and $\beta$ are acquired in SVM model.

At last, the test samples is normalized and input to the SVM model and the predicted result will be worked out. The relative errors are less than 5\%, shown in Fig. 3. 


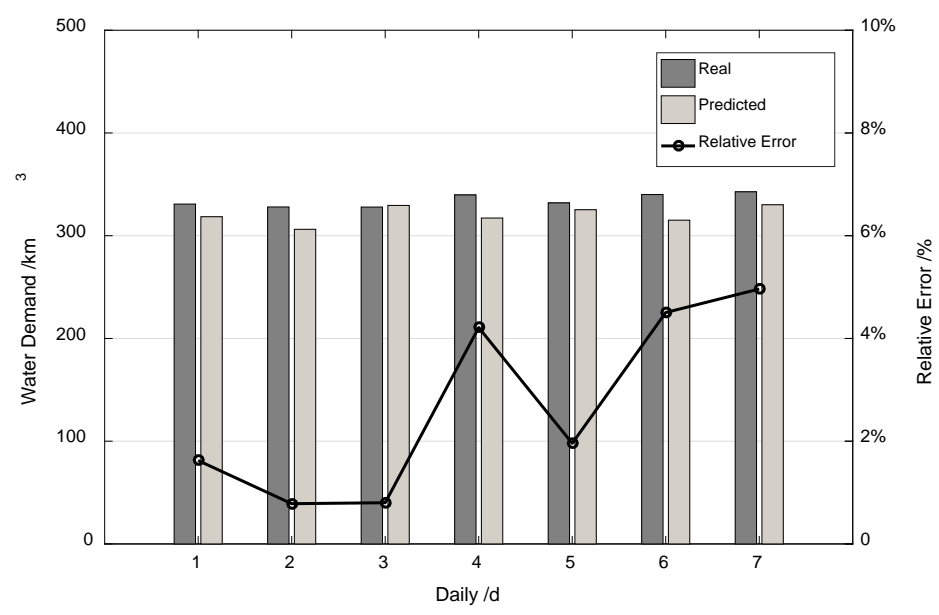

Fig. 3 The result of water usages prediction

For proving the effect of PSO-SVM, BP neural network and SVM model are employed to predicted the test samples and compared with PSO-SVM, shown in Table 4.

Table 4 Comparison of results and relative errors

\begin{tabular}{|c|c|c|c|c|c|c|c|}
\hline \multirow[b]{2}{*}{$\begin{array}{l}\text { Daily } \\
\text { /d }\end{array}$} & \multirow[b]{2}{*}{$\begin{array}{l}\text { Real } \\
/ \mathrm{km}^{3}\end{array}$} & \multicolumn{2}{|c|}{$\mathrm{BP}$} & \multicolumn{2}{|c|}{ SVM } & \multicolumn{2}{|c|}{ PSO-SVM } \\
\hline & & $\begin{array}{c}\text { Predicted } \\
/ \mathrm{km}^{3}\end{array}$ & $\begin{array}{c}\text { Error } \\
/ \%\end{array}$ & $\begin{array}{c}\text { Predicted } \\
/ \mathrm{km}^{3}\end{array}$ & $\begin{array}{c}\text { Error } \\
/ \%\end{array}$ & $\begin{array}{c}\text { Predicted } \\
/ \mathrm{km}^{3}\end{array}$ & $\begin{array}{c}\text { Error } \\
/ \%\end{array}$ \\
\hline $16-05-01$ & 330.80 & 318.49 & 3.72 & 328.17 & 0.79 & 325.43 & 1.62 \\
\hline 16-05-02 & 328.00 & 306.25 & 6.63 & 326.20 & 0.55 & 325.43 & 0.78 \\
\hline $16-05-03$ & 327.90 & 329.50 & 0.49 & 307.59 & 6.19 & 325.27 & 0.80 \\
\hline 16-05-04 & 339.70 & 317.21 & 6.62 & 310.04 & 8.73 & 325.39 & 4.21 \\
\hline 16-05-05 & 331.93 & 325.29 & 2.00 & 320.81 & 3.35 & 325.43 & 1.96 \\
\hline 16-05-06 & 340.08 & 315.13 & 7.34 & 317.30 & 6.70 & 324.76 & 4.50 \\
\hline $16-05-07$ & 342.73 & 330.14 & 3.39 & 316.40 & 7.41 & 324.77 & 4.96 \\
\hline
\end{tabular}

\section{Conclusions}

The PSO-SVM model predict daily water demand in city G. At first, parameters are selected by the PSO, the water demands trained samples are trained by SVM model. Finally using test samples predict water demands. The results demonstrate that the relative errors of real and predicted values is less than 5\% in PSO-SVM and more accurate than BP neural network and SVM model. Therefore, PSO-SVM has a good effect on prediction of daily water demand in city G.

\section{Acknowledgements}

This work is financially supported by the National Science and Technology Major Project (2014ZX07406003). The authors would like to thank the local water utility for providing historical data.

\section{References}

[1] Chen Y.C., Applications of time series analysis to water demand prediction, C. Computer Applications in Water Supply. (1988) 289-298. 
[2] Zhou S.L., Mcmahon T.A., Walton A., and Lewis J, Forecasting daily urban water demand: a case study of Melbourne, J. Journal of Hydrology. 236 (2000) 153-164.

[3] Jiang, Baiyi, D. Jin, and J. Gao, Comparing Study on Urban Daily Water Consumption Forecasting Model, J. Journal of Shenyang Jianzhu University. 24 (2008) 278-281.

[4] Zhao, M., Zhang, Q., Huang, Y., Gao, J., and Zhao, H, Water Consumption Prediction by Using SVM and Information Granular Time Series Regression, C. Numerical Methods for Partial Differential Equations. 8.2 (2012) 127-142.

[5] Li, H., Zhang, C., Miao, D., Wang, T., Feng, Y., and Fu, H., et al, Water Demand Prediction of Grey Markov Model Based on GM(1,1), C. International Conference on Mechatronics and Information Technology. (2016) 524-529.

[6] Rubio G., Pomares H., Rojas I., Herrera L.J., and Guillén A., Efficient Optimization of the Parameters of LS-SVM for Regression versus Cross-Validation Error, C. International Conference on Artificial Neural Networks. 5769 (2009) 406-415.

[7]Zhang L.B., Zhou C.G., Ming M.A., and XiaoHua A.L, Solutions of Multi-Objective Optimization Problems Based on Particle Swarm Optimization, J. Journal of Computer Research \& Development. 41 (2004) 1286-1291. 